Horizons philosophiques

\title{
Valeurs et interventions éducatives : cas du développement rural en Afrique subsaharienne
}

\section{Youssouf Sanogo}

Volume 12, numéro 2, printemps 2002

Valeurs et modernité

URI : https://id.erudit.org/iderudit/801211ar

DOI : https://doi.org/10.7202/801211ar

Aller au sommaire du numéro

Éditeur(s)

Collège Édouard-Montpetit

ISSN

1181-9227 (imprimé)

1920-2954 (numérique)

Découvrir la revue

\section{Citer cet article}

Sanogo, Y. (2002). Valeurs et interventions éducatives : cas du développement rural en Afrique subsaharienne. Horizons philosophiques, 12(2), 101-114.

https://doi.org/10.7202/801211ar d'utilisation que vous pouvez consulter en ligne.

https://apropos.erudit.org/fr/usagers/politique-dutilisation/ 


\section{VALEURS ET INTERVENTIONS ÉDUCATIVES : \\ CAS DU DÉVELOPPEMENT \\ RURAL EN AFRIQUE SUBSAHARIENNE}

\section{Introduction}

Les valeurs jouent un rôle prépondérant dans l'attitude et le comportement des individus. On pourrait dire que nous avons tous des valeurs, c'est-à-dire des principes profondément ancrés en nous qui déterminent ce que nous considérons comme bien, vertueux, acceptable ou mal. Les valeurs peuvent être individuelles ou propres à un groupe ou à une communauté donnée. Elles découlent des différents apprentissages que nous avons effectués, déterminent notre façon de juger ou d'agir, et façonnent nos réalisations. Ainsi, même les systèmes d'enseignement que nous concevons n'échappent pas à l'influence de nos valeurs. Celles-ci sont présentes et perceptibles à travers les finalités poursuivies.

Que ce soit à l'école ou dans les communautés, les interventions éducatives mettent en contact plusieurs valeurs. D'abord, chaque acteur (y compris l'enseignant) arrive avec les siennes, sa façon de voir et d'interpréter la réalité. Ensuite, à la multitude des valeurs individuelles s'ajoutent celles que véhicule le système d'enseignement. II faut dire que les valeurs du système d'enseignement ne sont pas nécessairement celles épousées par l'enseignant lui-même. Mais, à travers ses cours, l'enseignant contribue, de façon consciente ou pas, à véhiculer ces valeurs. Cela s'applique également aux situations de formation et d'animation.

Dans cet article, nous sortons du cadre classique des écoles pour nous situer dans le domaine de l'éducation pour le développement rural en Afrique au sud du Sahara. Dans cette partie du continent, par le fait de l'histoire, s'affrontent plusieurs valeurs éducatives découlant de la cohabitation de différents systèmes d'enseignement. Cela confère aux interventions éducatives, surtout celles menées dans une perspective de développement communautaire, un caractère plutôt complexe. 


\section{L'éducation en Afrique au sud du Sahara}

Pour mieux comprendre l'éducation en Afrique au sud du Sahara, il convient de s'attarder sur ce qui caractérise les différents systèmes d'enseignement qui la composent. Car ce sont, en fait, les valeurs véhiculées à travers les systèmes d'enseignement qui influencent dans une large mesure les attitudes et comportements des acteurs du développement rural, notamment les agents et les paysans. Ces valeurs, nous le verrons plus loin, sont inhérentes aux finalités éducatives poursuivies. Elles déterminent la philosophie éducative, le mode d'organisation des activités de formation, et le type de rapports à l'intérieur du système d'enseignement et entre celui-ci et les autres.

À la suite de Moumouni (1967), Nyéréré (1972), Ajayi (1986), Mazrui et Wagaw (1986) et Ki-Zerbo (1990), on pourrait dire que l'éducation en Afrique subsaharienne comporte une pluralité de tendances. Quatre tendances principales se dégagent : africaine, chrétienne, islamique et occidentale. La tendance africaine est celle qui existe traditionnellement en Afrique et les autres sont le résultat des différentes influences subies au cours de l'histoire. L'influence chrétienne date du IV ${ }^{\theta}$ siècle, l'influence islamique du VIIe siècle et celle venant de l'occident de la fin du XIX甲 siècle. Le degré d'influence des différentes tendances éducatives religieuses (chrétienne et islamique) diffère beaucoup d'une société à l'autre, d'un pays à l'autre ou d'une région à l'autre. Mais, celui de l'éducation de type occidental présente un aspect presque homogène partout en Afrique noire.

Chacune des tendances éducatives a son système d'enseignement. Mazrui et Wagaw (1986) classent les différents systèmes d'enseignement selon qu'ils ont des finalités explicites ou implicites sociocentriques, théocentriques, égocentriques ou écocentriques. Un système d'enseignement est dit sociocentrique lorsque sa finalité dernière est essentiellement de servir la société, de favoriser une vie sociale plus harmonieuse. Lorsque cette finalité est de servir Dieu, d'obtenir l'adhésion à une religion ou d'en affermir le respect des préceptes, il est dit théocentrique. II est considéré égocentrique quand son but est de permettre à chaque individu de s'accomplir, de libérer l'individualité et la créativité de chaque être. Le caractère écocentrique se réfère à la tendance à développer la curiosité, à cultiver et à encourager une compréhension globale écologique, c'est-à-dire la connaissance de la nature et de l'univers, indépendamment du service rendu à la société ou à Dieu. 
Cependant, il convient de souligner qu'il ne s'agit ici que de caractères dominants. II est toujours probable d'observer dans chaque système d'enseignement un chevauchement entre les différents caractères. Voyons, à présent, les différentes caractéristiques relevées par les différents auteurs pour chaque système d'enseignement.

\subsection{Système d'enseignement traditionnel africain}

Découlant fondamentalement des expériences et traditions africaines, le système d'enseignement traditionnel africain a précédé l'introduction des systèmes islamique, chrétien et occidental sur le continent africain. En dépit des influences subies, il continue à survivre, souvent au prix d'une certaine adaptation réciproque avec les autres.

Moumouni (1967, p. 13-14) soutient qu'au-delà de sa diversité, des particularités qui peuvent être observées ici et là, l'éducation en Afrique noire présente des traits généraux qui démontrent une communauté de cultures. D'abord, elle se caractérise par la grande importance qui lui est accordée en famille comme dans la communauté. Ensuite, elle se distingue par son caractère collectif et social, son lien intime avec la vie sociale sur le double plan matériel et spirituel, son caractère polyvalent aussi bien en ce qui concerne les objectifs visés que les moyens employés et par sa réalisation progressive et graduelle conformément aux étapes successives de l'évolution physique, psychique et mentale.

En raison de la grande importance que le système d'enseignement traditionnel africain accorde ainsi à la vie communautaire, Mazrui et Wagaw (1986) concluent qu'il est de tendance sociocentrique. Ils soutiennent que le but ultime des enseignements donnés n'est pas de donner nécessairement une indépendance intellectuelle à l'individu et de faciliter son auto-réalisation, mais de le préparer à remplir un rôle honorable et constructif dans la communauté (p. 40). L'intérêt commun ou collectif prend le pas sur l'individuel ou le particulier.

Cependant, parallèlement à l'éducation à la vie communautaire, chaque individu est également préparé à avoir une personnalité propre dans la société, à marquer une certaine individualité, à être responsable et avoir de l'autonomie dans ses prises de décisions et actions. D'ailleurs, à propos de la tendance sociocentrique, Mazrui et Wagaw (1986) nous mettent en garde contre une généralisation excessive. En effet, d'une ethnie à l'autre, il y a bien des différences 
qui se dégagent parfois dans les démarches éducatives. Au Nigeria, par exemple, le peuple lbo se montre relativement plus égocentrique que son voisin Hawsa qui, lui, est beaucoup plus tourné vers le sociocentrisme. En outre, dans les communautés africaines, il n'est pas rare d'observer une certaine rivalité entre individus ou entre familles. Toutefois, en dépit de la préparation à exprimer une certaine individualité dans la communauté, et de la présence des cas de rivalité et d'égocentrisme sur le continent, la tendance dominante du système d'enseignement traditionnel africain reste le sociocentrisme.

Au-delà du caractère évident de son sociocentrisme, le système d'enseignement traditionnel africain présente aussi des tendances écocentrique et théocentrique. En fait, il n'est pas aisé de départir ces deux tendances, tant l'écocentrisme traditionnel débouche sur des considérations divines ou religieuses. Mazrui et Wagaw (1986) expliquent que l'écocentrisme traditionnel africain est dominé, non pas par une curiosité résolument orientée vers la compréhension et le contrôle de la nature et de l'univers, mais plutôt par un certain souci pour l'environnement. II apparait que l'Africain essaie de comprendre l'écologie pour mieux vivre en harmonie avec son environnement. $\mathrm{Ce}$ souci de l'écologie découle des différentes croyances traditionnelles africaines, basées essentiellement sur l'animisme et recherchant constamment une certaine harmonie entre l'humain et la nature, les vivants et les morts, le visible et l'invisible. Dans ces croyances, l'on considère que l'homme, la nature et Dieu se pénètrent l'un et l'autre. D'où le caractère socialisant de la morale enseignée dans les communautés.

Par ailleurs, le système d'enseignement traditionnel africain est marqué par une dominance de la tradition orale. Les écrits sont rares et cela nous paraît une de ses faiblesses. Les connaissances sont généralement gardées en mémoire d'homme, entravant ainsi leur transmission systématique aux autres. Finalement, comme l'a dit Amadou Hampaté Bah (cité dans Ki-Zerbo, 1990, p. 52), en Afrique, "lorsqu'un vieillard meurt, c'est une bibliothèque qui brûle».

Quant à l'organisation de l'enseignement, l'on peut noter chez Moumouni (1967), Mazrui et Wagaw (1986), Ajayi (1986), Erny (1987) et Itoua et al. (1988) que le système traditionnel africain n'est pas formaliste. II est plutôt non formel en ce sens que les cours ne sont pas dispensés comme à l'école formelle. II n'y a pas d'écoles construites pour l'enseignement, pas d'horaires particuliers pour telle ou telle matière et pas de système de classement des élèves selon les 
performances. L'apprentissage se fait au jour le jour en famille comme dans la communauté : l'école se confond avec la vie et l'éducation avec la socialisation. Cependant, l'enseignement n'est pas informel pour autant; c'est-à-dire occasionnel et hasardeux. Dans la pratique, comme l'explique Moumouni (1967), l'éducation traditionnelle présente un caractère global et suit une certaine structure, non écrite certes, mais connue de tous. Elle est la responsabilité non seulement des parents, mais aussi des autres membres de la famille 1 et de la communauté tout entière. L'enfant est généralement traité comme un disciple qui doit obéissance et respect à ses parents et aînés qui, eux, ont la responsabilité de l'éduquer selon les valeurs sociales jusqu'à ce qu'il atteigne l'âge adulte. La formation ainsi donnée fait constamment appel au travail manuel comme au travail intellectuel; elle alterne théories et pratiques.

\subsection{Systèmes d'enseignement islamique et chrétien}

Ces deux systèmes, qui furent introduits en Afrique au cours de I'histoire, se rapprochent beaucoup de par les finalités poursuivies (Ajayi, 1986 et Mazrui et Wagaw, 1986). Tous les deux sont dominés par la tendance théocentrique. Ils mettent l'emphase sur la religion : l'Islam pour les musulmans et le Christianisme pour les chrétiens. Tous visent surtout à cultiver le respect de la volonté de Dieu et à développer un esprit et un comportement religieux chez les adeptes.

Ajayi (1986) relève notamment que le système d'enseignement islamique est axé non seulement sur l'Islam, mais aussi sur la langue et la culture arabes. Quant au système d'enseignement chrétien, en plus de sa mission traditionnelle d'étendre la religion chrétienne, il s'est avéré lui aussi un facteur d'expansion des langues et cultures occidentales. L'auteur trouve d'ailleurs que l'expansion missionnaire a été le précurseur de la conquête et de la domination coloniales de l'Europe en Afrique. Le système de formation chrétien a aussi été une dimension de la colonisation.

Ajayi (1986) et Mazrui et Wagaw (1986) s'accordent pour dire que les systèmes d'enseignement chrétien et islamique sont élitistes. Le caractère élitiste s'explique par le fait que chacun des deux systèmes met constamment l'accent sur la valorisation de la religion dont il est issu et s'adonne à un dénigrement des croyances et de certains

1. La famille est mentionnée ici au sens africain. Elle ne se limite pas aux seuls parents biologiques, aux frères et sœurs. Elle s'étend aussi aux cousins, tantes, oncles et grands-parents. 
aspects du mode de vie africain, considérés comme rétrogrades et païens. Ainsi, dans le quotidien, il arrive que certains adhérents à ces différentes religions se distinguent par leur mépris pour les pratiques traditionnelles africaines au profit de la foi monothéiste chrétienne ou musulmane. Les plus instruits accèdent facilement à des positions religieuses qui leur confèrent des avantages et une certaine distinction sociale. De cette façon, on devient, entre autres, imam, muezzin, mwalime, cheikh, shérif chez les musulmans, ou encore évêque, prêtre, pasteur, entre autres, chez les chrétiens.

Les deux systèmes présentent également des caractères sociocentriques et écocentriques. Mais, ceux-ci sont surtout dominés par la volonté de servir Dieu et de promouvoir un esprit et un comportement religieux. Ainsi, le caractère sociocentrique est dominé par des principes relevant des préceptes du Coran chez les musulmans ou de la Bible chez les chrétiens. Finalement, seuls les fidèles sont concernés par les formes de socialisation professées. Le reste de la population, encore attaché à d'autres croyances, se trouve de facto exclu. De même, le caractère écocentrique est empreint de religion. La curiosité manifestée chez les adeptes s'inscrit dans le cadre religieux et ne devrait pas remettre en cause ce qui est dit dans le livre saint.

Par ailleurs, au delà de ces caractéristiques générales des systèmes d'enseignement religieux monothéistes, il est important de signaler que des congrégations chrétiennes se sont distinguées par les services sociaux rendus aux populations. Dans certaines régions, notamment en Afrique de l'Ouest, des missions chrétiennes ont ouvert des écoles, des dispensaires, et des ateliers de formation professionnelle dans les domaines tels que la menuiserie et la couture. Ces centres étaient ouverts à toute la population, y compris les habitants qui n'étaient pas de foi chrétienne. On pourrait d'ailleurs mettre au crédit des missions chrétiennes la formation de la plupart des premiers cadres africains, dont certains n'étaient pas chrétiens.

\subsection{Système d'enseignement de type occidental}

Introduit en Afrique subsaharienne par le biais de la colonisation, le système d'enseignement de type occidental a d'abord été un véritable outil d'assimilation des élèves africains et de dénigrement des pratiques et croyances africaines (Moumouni, 1967; Ajayi 1986; Mazrui et Wagaw, 1986). Certes, les démarches différaient selon la métropole, mais le but visé était le même : la colonisation de l'Afrique 
et la poursuite des intérêts du pays colonisateur. Dans cette entreprise, les puissances coloniales ont dû parfois collaborer avec les missions chrétiennes, mais la finalité poursuivie ne changeait pas pour autant.

Ce qui caractérise l'enseignement de type occidental, ce sont surtout ses tendances égocentrique et écocentrique (Mazrui et Wagaw, 1986). Son égocentrisme se manifeste par la grande importance accordée à l'accomplissement de l'individu et à son propre épanouissement. Quant à son écocentrisme, il se présente non pas comme un souci pour l'écologie, mais comme une curiosité pour comprendre cette dernière, la maîtriser, voire la transformer et la contrôler de façon à favoriser l'accomplissement de soi, l'épanouissement socio-économique. Cette philosophie qui caractérise le système d'enseignement de type occidental le distingue des autres types de formation donnée en Afrique au sud du Sahara. Et toute la philosophie éducative qui le sous-tend semble rimer avec la vision égocentrique et la curiosité écologique.

L'enseignement de type occidental présente un caractère formel. Contrairement au système d'enseignement traditionnel africain, l'école ne se confond pas avec la vie de tous les jours. L'éducation non plus ne se confond pas avec la socialisation telle que prônée dans le système traditionnel africain. Les cours sont dispensés dans les écoles qui, elles, sont classées en fonction des paliers d'enseignement (Moumouni, 1967; Diambomba, 1980 et Weva, 1982). L'enseignement est la responsabilité des maîtres et professeurs, qui forment selon des programmes standards, repartis en domaines et disciplines et étalés sur des périodes déterminées. Les apports des familles et sociétés africaines y sont relativement minimes. Les apprenants sont formés à développer une certaine logique plus ou moins scientifique qui leur permette d'appréhender et de contrôler leur cadre de vie. Contrairement à l'éducation traditionnelle, le plus souvent, tout ce qui échappe à la logique scientifique (tels que le monde de l'invisible et celui des ancêtres) est banalisé.

Le principal reproche fait à l'endroit de l'école formelle en Afrique noire (Nyéréré, 1972; Mazrui et Wagaw, 1986; Devisse, 1986; Tettekpoe, 1988 et Ki-Zerbo, 1990) est d'avoir éloigné les enfants des normes et valeurs africaines. L'école les assimile à la culture occidentale, principalement aidée en cela par le fait que la langue d'enseignement est, en général, une langue étrangère au milieu : celle de l'ancienne métropole. Elle cultive le mépris des traditions et 
favorise l'élitisme et l'individualisme dans une société où la vie communautaire est une tradition.

Cette tendance, qui caractérise l'enseignement formel, semble avoir développé chez certains africains une attitude négativiste vis-àvis de la société traditionnelle (Nyéréré, 1972, p. 24). Ces derniers ont tendance à remettre en cause les traditions et à considérer les villageois comme dépassés, anachroniques. De leur côté, les villageois, plus traditionalistes, tiennent beaucoup au respect des normes sociales existantes. Parfois, exaspérés par le zèle et l'attitude contestataire des scolarisés, certains traditionalistes n'hésitent pas à rappeler à ces derniers l'adage qui dit : «le tronc d'arbre a beau séjourner dans l'eau, il ne deviendra jamais un crocodile, même s'il pouvait bien noircir comme lui». En d'autres termes : l'Africain a beau fréquenter l'école, il ne deviendra jamais un Occidental, même s'il pouvait bien s'occidentaliser. Le roman de Badian (1972) reflète les scènes typiques de cet antagonisme entre traditionalistes et modernistes.

Toutefois, il convient de préciser que l'attitude négativiste vis-àvis des us et coutumes africains n'est pas l'apanage de tous les scolarisés. Bien des scolarisés saisissent la relativité des enseignements reçus à l'école.

\subsection{Influences des différents systèmes d'enseignement sur les acteurs de développement rural}

Aux différentes finalités éducatives correspondent des valeurs qui diffèrent selon que l'on suit l'un ou l'autre système d'enseignement. Au plan social, la cohabitation des quatre types d'enseignement a plutôt créé des interactions entre différentes sortes de philosophie éducative. Cela a laissé un certain impact sur les populations (Diawara, 1997; Mc Laughin, 1997; Der Geest, 1997), à tel point que l'Africain subsaharien de nos jours présente, en général, un caractère intégrant à la fois des caractéristiques éducatives de l'enseignement traditionnel africain, de celui de type occidental et, le plus souvent, de la religion monothéiste (Islam ou Christianisme) qu'il pratique². II est effectivement difficile d'imaginer un Africain subsaharien ayant grandi en Afrique, si chrétien ou musulman soit-il, qui n'ait pas subi plus ou moins les influences des enseignements traditionnels. Abondant dans le même sens, Erny (1987) fait deux constatations :

2. A présent, il existe des Africains qui ne pratiquent ni l'Islam, ni le Christianisme, mais seulement les croyances traditionnelles. 
1. Nulle part il n'est plus possible de retrouver l'éducation traditionnelle à l'état pur, entièrement préservée d'influences étrangères. Mais les altérations qu'elle subit l'affectent à des degrés et à des niveaux très différents selon les ethnies, les régions, les familles et les individus.

2. Nulle part elle ne s'est complètement effacée pour laisser la place nette à une éducation de type occidental moderne. Même dans les milieux les plus européanisés il est toujours possible d'en retrouver certains éléments, et le plus souvent elle continue à former la toile de fond des apports éducatifs que l'enfant reçoit de sa famille et de son milieu (p. 270).

Dans cette situation, on ne peut que parler de prédominance éducative. En général, les influences des types d'enseignement sont perceptibles même au niveau des attitudes, des comportements et des modes de pensée. Ces derniers varient le plus souvent d'une personne à l'autre, selon qu'elle a reçu une éducation à prédominance islamique, chrétienne, occidentale ou traditionnelle. Ainsi, en raison de la différence de prédominance éducative, les paysans et les agents ne présentent pas le même profil.

D'un côté, les agents de développement, tout comme les autres développeurs ${ }^{3}$ d'ailleurs, reçoivent une éducation à prédominance occidentale. Ils sont formés dans les écoles formelles. Ayant ainsi bénéficié d'un enseignement à dominance égocentrique, avec un écocentrisme à curiosité écologique, ils sortent en principe avec une certaine logique, tirée dans une large mesure de la formation reçue. De l'autre côté, les paysans reçoivent une éducation à prédominance africaine. Ils reçoivent alors un enseignement à prédominance sociocentrique, avec un écocentrisme à souci écologique. Vivant au village et pratiquant des occupations traditionnelles d'agriculture, d'élevage, de pêche et autres, ils se caractérisent par leur vision globale intégrant à la fois la nature et la société, les morts et les vivants, le visible et l'invisible.

\section{La complexité des interventions éducatives pour le développement rural}

La complexité des interventions éducatives est à l'image de celle de l'ensemble des actions de développement entamées en direction

3. Par "développeurs", nous désignons tous ceux qui, autres que les populations bénéficiaires, sont impliqués dans la planification et l'exécution des actions de développement : décideurs de politiques de développement, chercheurs et agents de développement, etc. 
du monde rural. Les valeurs jouent un rôle prépondérant dans les différents facteurs qui la caractérisent. Nous en retenons trois facteurs essentiels : les différences de prédominances éducatives des acteurs de développement, l'écart entre les valeurs prônées par le système d'intervention et les attentes des bénéficiaires, et la tendance à s'accrocher à ses valeurs, sa vision et sa logique.

D'abord, en raison des différences de prédominances éducatives, agents et paysans n'entament souvent pas les actions de développement avec les mêmes visions, les mêmes logiques. Ainsi, selon Sardan et Paquot (1991, p.17), autour des actions de développement, deux mondes entrent en contact, deux cultures, deux univers de significations, deux systèmes d'interprétations, bref deux systèmes de sens. En conséquence, on assiste à des malentendus, voire des contradictions, autour des actions entamées; ce qui rend les interventions pour le développement rural (y compris les interventions éducatives) en Afrique subsaharienne complexes, c'est-à-dire imprévisibles à travers les plans élaborés.

Ensuite, il faut compter avec les valeurs véhiculées à travers les systèmes d'intervention. Par exemple, dans une étude de cas au Mali, dans la zone encadrée par la Compagnie Malienne de Développement de Textiles (CMDT) (Sanogo, 2001), l'on se rend compte de l'écart entre les finalités poursuivies à travers les activités d'alphabétisation fonctionnelle et les attentes des paysans bénéficiaires. D'un côté, contrairement à la politique de développement global officiellement annoncée, de facto le programme d'alphabétisation semble être conçu pour assurer surtout les intérêts économiques de la CMDT. Il s'agit de former les producteurs agricoles de façon à promouvoir la culture et la commercialisation du coton, dont ladite compagnie est la première exportatrice, négligeant du coup les préoccupations socio-économiques des producteurs agricoles. Les valeurs véhiculées se distinguent par un égocentrisme dominé par la recherche du seul épanouissement économique de la CMDT, même au détriment des producteurs agricoles.

De l'autre côté, sous le poids de la situation de crise socioéconomique créée par la politique de monopole et de monoculture de la CMDT, certes les producteurs reconnaissent l'importance des cours liés au coton, devenu leur seule source de revenu, mais ils souhaitent également une plus grande variation du contenu de la formation donnée. Entre autres, ils aimeraient avoir aussi des cours relatifs à d'autres cultures agricoles, à l'élevage, au petit commerce, de façon à 
avoir plus de liberté de choix, plus d'autonomie, et pouvoir développer des activités qui leur semblent plus socialement et économiquement viables. D'une manière générale, les valeurs recherchées par les producteurs se révèlent d'ordre sociocentrique. Elles font ressortir une volonté d'établir un équilibre entre le social et l'économique au village, dans l'intérêt global des populations.

L'écart entre les attentes des producteurs agricoles et les valeurs privilégiées dans la politique d'intervention de la CMDT a entraîné des frustrations au niveau des populations paysannes. Des revendications, des jacqueries et le boycott de la culture de coton s'ensuivirent, faussant du coup tous les plans de développement "savamment" conçus par la hiérarchie politico-technocratique de la CMDT. La conséquence économique directe est la chute de la production du coton, faisant perdre au Mali son rang de deuxième producteur africain de ce produit; ce sont également la récession économique que connaît présentement ce pays et la vague d'arrestations pour délinquance financière de certains hauts cadres de la CMDT.

Enfin, au-delà de la différence de prédominances éducatives entre agents et paysans, de l'écart entre les valeurs prônées à travers le système d'intervention et les attentes des populations, il y a aussi la tendance des individus eux-mêmes à s'accrocher à leurs valeurs, à leurs visions, à leurs logiques. Le danger est que chacun devienne alors prisonnier de ses propres valeurs, résistant ainsi aux propositions venant des autres. Puisqu'une intervention éducative met en interaction plusieurs acteurs, chacun y arrive avec ses valeurs et interprète les sujets abordés à partir de sa logique. II en découle des malentendus et des contradictions qui ajoutent leur lot de complexité aux interventions éducatives.

Comment aborder la complexité des situations d'intervention éducative en tant qu'intervenant? À ce propos, nous nous en tenons aux résultats de la recherche menée dans la zone CMDT du Mali (Sanogo, 2001). D'abord, il convient de savoir qu'en raison de leur complexité, l'appréhension des situations d'intervention en milieu rural requiert une approche plus globale pour mieux prendre en compte les différents valeurs, facteurs et interactions en jeu. Ensuite, en raison de cette complexité, il n'existe pas une seule méthode de gestion des actions en milieu rural. Enfin, il importe de privilégier la viabilité par rapport au contexte d'application comme critère de validité d'une méthode, d'une décision ou d'une action. Toute démarche devra respecter le principe d'ouverture envers l'ensemble des acteurs con- 
cernés. Et chacun devra accepter de remettre en cause toute valeur, toute vision ou toute logique non viable pour l'amélioration des situations problématiques du moment, et non socialement et culturellement acceptables par l'ensemble des acteurs.

\title{
4. Conclusion
}

Que ce soit dans le domaine du développement rural ou à l'école, les interventions éducatives occasionnent des situations où plusieurs valeurs viennent en contact. De cette diversité des valeurs et des interactions entre acteurs émergent, dans une large mesure, des situations d'intervention éducative complexes. Et cette complexité, si elle n'est pas gérée de façon convenable, pourrait entraîner la dérive de l'ensemble des activités d'intervention. D'où l'importance pour l'enseignant, le formateur ou l'animateur de s'ouvrir aux autres acteurs concernés, d'essayer de comprendre leurs valeurs, afin de mieux intégrer celles-ci dans les activités d'enseignementapprentissage. Cette tâche n'est point facile ni pour l'enseignant, ni pour les apprenants, car il exige de la part de chacun une certaine capacité de prendre du recul par rapport aux siennes.

En raison du caractère évolutif et imprévisible des situations d'intervention éducative, on ne saurait parler de la méthode de gestion des activités d'enseignement-apprentissage. II appartient à l'enseignant de réfléchir et d'agir comme un véritable professionnel, de prendre des initiatives et d'essayer des alternatives, dans le respect des caractéristiques des acteurs concernés. Toutefois, dans les démarches, il est important que chaque acteur (y compris les apprenants) respecte les principes d'ouverture, de dialogue, de remise en cause continue de ses propres visions, afin de déterminer ensemble avec les autres des décisions et des actions qui soient viables pour le contexte, et acceptables pour tous.

\author{
Youssouf Sanogo \\ Stagiaire post doctoral \\ Faculté d'éducation \\ Université de Sherbrooke
}




\section{Références}

Ajayi, J.F.A. (1986), «L'éducation dans l'Afrique contemporaine : historique et perspective", Le processus d'éducation et l'historiographie en Afrique, (documents de travail et compte rendu du colloque organisé par l'Unesco à Dakar, Sénégal, du 25 au 29 janvier 1982), p.11-22, Paris, Unesco.

Badian, S. (1972), Sous l'orage (Kany), suivi de La mort de Chaka, Paris, Présence Africaine.

Der Geest, S.V. (1997), «Money and Respect: The Changing Value of Old Age in Rural Ghana," Africa, vol.67, no 4, p.534-559.

Devisse, J. (1986), «L'éducation, l'enseignement et la formation en Afrique : évolution historique de 1930 à 1980, "Le processus d'éducation et l'historiographie en Afrique, (documents de travail et compte rendu du colloque organisé par l'Unesco à Dakar, Sénégal, du 25 au 29 janvier 1982), p. 97-105, Paris, Unesco.

Diambomba, M. (1980), La réforme scolaire au Mali : essai d'analyse des facteurs qui atténuent ses résultats, no 15, Québec, Université Laval, Faculté des sciences de l'éducation, Projet Ouest-africain de formation à la recherche évaluative en éducation.

Diawara, M. (1997), “"Dieu d'eau”, eau du barrage. Les populations du plateau Dogon face aux contraintes : pluviométrie, terre et démographie," Africa, vol. 67, no 4, p. 602-624.

Erny, P. (1987), L'enfant et son milieu en Afrique noire : essais sur l'éducation traditionnelle, Paris, Éditions L'Harmattan.

Itoua, F. et al. (1988), Famille, enfant et développement en Afrique, Paris, Unesco.

Ki-Zerbo, J. (sous la direction de) (1990), Éduquer ou périr, Paris, UnicefUnesco.

Mazrui, A. A. et T. Wagaw (1986), «Vers une décolonisation de la modernité: éducation et conflits culturels en Afrique de l'est,» Le processus d'éducation et l'historiographie en Afrique, (documents de travail et compte rendu du colloque organisé par l'Unesco à Dakar, Sénégal, du 25 au 29 janvier 1982), p. 35-64, Paris, Unesco.

Mc Laughin, F. (1997), "Islam and Popular Music in Senegal : The Emergence of a "New Tradition"," Africa, vol. 67, no 4, p. 560-581.

Moumouni, A. (1967), L'éducation en Afrique, Paris, François Maspero.

Nyéréré, J. (1972), Indépendance et éducation, Yaoundé, Cameroun, Éditions CLE.

Sanogo, Y. (2001), Principes directeurs de la conception et de la mise en œuvre des programmes de formation continue des responsables villageois : mise à contribution des représentations des acteurs de terrain : cas de Konoko, village de la zone cotonnière de la compagnie malienne de développement de textiles (CMDT), République du Mali, Thèse de doctorat, Technologie éducative, Département d'études sur l'enseignement et l'apprentissage, Faculté des sciences de l'éduca- 
tion, Université Laval, Québec, Juin 2001.

Sardan, J. P. O. D. et E. Paquot (1991), D'un savoir à l'autre : les agents de développement comme médiateurs, Paris, GRET.

Tettekpoe, D. A. «La famille traditionnelle togolaise face à un développement endogène et centré sur l'homme africain", in Itoua, F. et al.

(1988). Famille, enfant et développement en Afrique, p. 22-38, Paris, Unesco.

Weva, K. W. (1982), Étude critique de l'application de la théorie de l'éducation pour l'auto-dépendance au développement rural en Tanzanie, no 30, Québec, Université Laval, Faculté des sciences de l'éducation, Département d'administration et politique scolaires. 